\title{
Isotropic hypoellipticity and trend to the equilibrium for the Fokker-Planck equation with high degree potential
}

\author{
Frédéric Hérau
}

\begin{abstract}
We consider the Fokker-Planck equation with a confining or anti-confining potential which behaves at infinity like a possibly high degree homogeneous function. Hypoellipticity techniques provide the well-posedness of the weakCauchy problem in both cases as well as instantaneous smoothing and exponential trend to equilibrium. Lower and upper bounds for the rate of convergence to equilibrium are obtained in terms of the lowest positive eigenvalue of the corresponding Witten Laplacian, with detailed applications.
\end{abstract}

\section{Introduction.}

We present here a joint work [12] with Francis Nier, from the University of Rennes. We consider the Fokker-Planck equation in $\mathbb{R}_{x, v}^{2 d}$

$$
\left\{\begin{array}{l}
\partial_{t} f+\frac{v}{m} \cdot \partial_{x} f-\frac{1}{m} \partial_{x} V(x) \cdot \partial_{v} f-\gamma_{0} \partial_{v} \cdot\left(\frac{1}{m \beta} \partial_{v}+v\right) f=0 \\
f(x, v, t=0)=f_{0}(x, v)
\end{array}\right.
$$

with a "high degree potential", $V(x) \simeq \pm|x|^{2 n}$ as $x \rightarrow \infty$ with $n \geq 1$ (possibly $n>1 / 2)$. The physical constants denoted by $m, \beta$ and $\gamma_{0}$ are respectively the particle mass, the inverse temperature $\beta=\frac{1}{k T}$ where $k$ is the Boltzmann constant and the friction coefficient.

For $V>0$ at infinity, all steady states in $\mathcal{S}^{\prime}\left(\mathbb{R}^{2 d}\right)$ are proportional to the Maxwellian

$$
M(x, v)=e^{-\beta p(x, v)},
$$

where $p(x, v)$ denotes the classical Hamiltonian $\frac{1}{2} m v^{2}+V(x)$.

Meanwhile, it is clear that for $V<0$ at infinity, the equation (1) has no steady state in $\mathcal{S}^{\prime}\left(\mathbb{R}^{2 d}\right)$ except 0 . Note that although the Cauchy problem (1) might not be well-posed for $\gamma_{0}=0$, it is (and this will be checked further) for $\gamma_{0}>0$ and $T>0$. 
We are interested in accurate estimates of the rate of convergence to equilibrium with respect to $m, \gamma_{0}, \beta$ and $V$. This question was raised by C. Villani in [28] and is related to the physically relevant range of time for general kinetic models. In the case of a negative potential, this can be interpreted as the life time of metastable states (cf. [24]).

The trend to equilibrium was studied by several authors at least for some specific potentials. Bouchut and Dolbeault obtained it in [1] by some compactness arguments and as well as decay for a nonlinear Vlasov-Fokker-Planck problem with a free energy decay argument. Talay got some implicit exponential decay [26][27] by studying directly the stochastic process. In [2], Villani and Desvillettes gave an explicit polynomial decay for some decaying perturbations of quadratic potentials with the help of logarithmic Sobolev inequalities. In the more complex situation of a chain of anharmonic oscillators coupled to heat baths, Eckmann et al. proved in [5] and [4] the existence and uniqueness of a steady state by the isotropic hypoellipticity techniques that they introduced for such problems but resolvent and spectral estimates are missing in order to get exponential trend to the steady state. More recently, Rey-Bellet and Thomas in [21][22][23] have obtained implicit exponential return to equilibrium in the mean for chains of anharmonic oscillators by going back to the stochastic approach and by introducing some Lyapunov functional.

\section{Statement of the main theorem}

We first introduce the isotropic Sobolev chain (isotropy means here and in the sequel that the phase-space position variables $(x, v)$ and the associated frequency variables play the same role):

$$
H^{s, s}\left(\mathbb{R}^{2 d}\right)=\left\{G \in \mathcal{S}^{\prime}\left(\mathbb{R}^{2 d}\right),\left(1+D_{x}^{2}+D_{v}^{2}+x^{2}+v^{2}\right)^{s / 2} G \in L^{2}\left(\mathbb{R}^{2 d}\right)\right\}
$$

with $s \in \mathbb{R}, D_{x, v}=\frac{1}{i} \partial_{x, v}$, and for which we have $\mathcal{S}^{\prime}\left(\mathbb{R}^{2 d}\right)=\cup_{s \in \mathbb{R}} H^{s, s}\left(\mathbb{R}^{2 d}\right)$ and $\mathcal{S}\left(\mathbb{R}^{2 d}\right)=$ $\cap_{s \in \mathbb{R}} H^{s, s}\left(\mathbb{R}^{2 d}\right)$. In fact we shall use the following spaces deduced from the preceding ones after multiplication by the $\mathcal{C}^{\infty}$-function $M^{1 / 2}$ such as

$$
M^{1 / 2} \mathcal{S}^{\prime}\left(\mathbb{R}^{2 d}\right) \stackrel{\text { def }}{=}\left\{g \in \mathcal{D}^{\prime}\left(\mathbb{R}^{2 d}\right), M^{-1 / 2} g \in \mathcal{S}^{\prime}\left(\mathbb{R}^{2 d}\right)\right\},
$$

and $M^{1 / 2} H^{s, s}\left(\mathbb{R}^{2 d}\right)$.

When $V$ is positive at infinity, $M^{1 / 2} \in \mathcal{S}$ and we denote by $M_{0}$ the $L^{1}$ normalized Maxwellian. Then $\int f=<M_{0}^{-1 / 2} f, M_{0}^{1 / 2}>_{\mathcal{S}^{\prime}, \mathcal{S}}$ is well defined for $f \in M^{1 / 2} \mathcal{S}^{\prime}$. We introduce the following operators for $f \in M^{1 / 2} \mathcal{S}^{\prime}$

$$
M^{1 / 2} \mathcal{S}^{\prime} \ni f \longmapsto\left(\left(\int f\right) M_{0}\right)_{ \pm}= \begin{cases}\left(\int f\right) M_{0} & \text { if } V>0 \text { near } \infty \\ 0 & \text { if } V<0 \text { near } \infty .\end{cases}
$$

The precise assumptions on the potential $V$ are

Hypothesis 1. The potential $V$ is a $\mathcal{C}^{\infty}$ function and there exist $n \geq 1$ (possibly $n>1 / 2$ ) and there are positive constants $C_{1}$ and $C_{\alpha}$ for all $\alpha \in \mathbb{N}^{d}$ so that for all $x \in \mathbb{R}^{d}$,

$$
\left|\partial_{x}^{\alpha} V(x)\right| \leq C_{\alpha}\left(1+\langle x\rangle^{2 n-\min \{|\alpha|, 2\}}\right) \quad \text { and } \quad\left|\partial_{x} V(x)\right| \geq C_{1}^{-1}\langle x\rangle^{2 n-1}-C_{1}
$$


Along the paper $C(V)$ will denote any finite family seminorms of $V$ which can be chosen positive and an index in $C(V)_{\text {index }}$ will possibly specify on which the choice of this family depends. We introduce the scaled potential

$$
V_{\beta}(x)=\beta V\left(\beta^{-1 / 2 n} x\right)
$$

and the associated Witten Laplacian on 0-forms

$$
A_{0}\left(V_{\beta}\right)=\left(-\partial_{x}+\frac{1}{2} \partial_{x} V_{\beta}\right) \cdot\left(\partial_{x}+\frac{1}{2} \partial_{x} V_{\beta}\right)
$$

Under the above assumptions, this operator after Friedrichs extension is self-adjoint, non-negative and with a compact resolvent (about this subject see the comments at the end of the paper). We introduce its smallest nonzero eigenvalue:

$$
\omega_{1}\left(V_{\beta}\right)=\min \sigma\left(A_{0}\left(V_{\beta}\right)\right) \backslash\{0\} .
$$

For convenience we define for $\nu \in \mathbb{N}$ and $R \in \mathbb{R}$ the function $Q_{R}$ on $\left(\mathbb{R}_{+}^{*}\right)^{\nu}$ by $Q_{R}\left(t_{1}, \ldots, t_{\nu}\right)=\prod_{j=1}^{\nu}\left(t_{j}+t_{j}^{-1}\right)^{R}$.

Theorem 2.1. Under Hypothesis 1 ( $n>1 / 2)$ and for $\gamma_{0}, \beta, m>0$, we have:

1) The Cauchy problem (1) is well-posed for $t \geq 0$ in $M^{1 / 2} \mathcal{S}^{\prime}\left(\mathbb{R}^{2 d}\right)$. Moreover the solution $f(t)$ belongs to $M^{1 / 2} \mathcal{S}\left(\mathbb{R}^{2 d}\right)$ for $t>0$.

2) There exists a real $\tau=\tau\left(\beta, \gamma_{0}, m\right)>0$ and for any $s \geq 0$ two constant $c_{s}>0$ and $R_{s}>0$ so that the estimate

$$
\left\|f(t)-\left(\left(\int f_{0}\right) M_{0}\right)_{ \pm}\right\|_{M^{1 / 2} H^{s, s}} \leq c_{s} Q_{R_{s}} e^{-\tau t}\left\|f_{0}\right\|_{M^{1 / 2} H^{-s,-s}}
$$

holds for all $f_{0} \in M^{1 / 2} H^{-s,-s}\left(\mathbb{R}^{2 d}\right)$ with $Q_{R_{s}}=Q_{R_{s}}\left(C(V)_{s}, \sqrt{m} \gamma_{0}, \beta, t, \tau, m\right)$ and for $s=0, Q_{R_{0}}=Q_{R_{0}}\left(C(V), \sqrt{m} \gamma_{0}, \beta, t, \tau\right)$.

3) In part 2), we can take

$$
\tau\left(\beta, \gamma_{0}, m\right)=\tau_{1}\left(\beta, \gamma_{0}, m\right) \stackrel{\text { def }}{=} \frac{\gamma_{0} \min \left\{1, \omega_{1}\left(V_{\beta}\right)\right\}}{64\left(5+3 \gamma_{0} \sqrt{m} \beta^{\frac{n-1}{2 n}}+3 C_{V_{\beta}}\right)^{2}}
$$

where $C_{V_{\beta}}^{2}=\max \left\{\sup \left(\left(H e s s V_{\beta}\right)^{2}-\left(\frac{1}{4}\left(\partial_{x} V_{\beta}\right)^{2}-\frac{1}{2} \Delta V_{\beta}\right) I d\right), 0\right\}$.

4) There exists a constant $c>0$, so that any $\tau$ satisfying part 2) is bounded by

$$
\tau \leq c \sqrt{\omega_{1}\left(V_{\beta}\right)} \log \left[Q_{R_{0}}\left(C(V), \sqrt{m} \gamma_{0}, \beta, \omega_{1}\left(V_{\beta}\right)\right)\right] .
$$

For a probability measure $f_{0}$ and in the case $V>0$ near $\infty$, we get as a corollary the exponential decay of the relative entropy with respect to the normalized Maxwellian $M_{0}$

$$
H\left(f(t) \mid M_{0}\right)=\int f(t) \log \left(\frac{f(t)}{M_{0}}\right) d x d v \quad(t>0) .
$$


Corollary 2.2. There are two constants $c^{\prime}>0$ and $R^{\prime}>0$ so that for all probability measure $f_{0} \in M^{1 / 2} \mathcal{M}_{b}\left(\mathbb{R}^{2 d}\right)$ the solution $f(t)$ to the Fokker-Planck equation 1 satisfies

$$
\forall t>0, H\left(f(t) \mid M_{0}\right) \leq c^{\prime} Q_{R^{\prime}}\left(C(V), \sqrt{m} \gamma_{0}, \beta, t, \tau_{1}\right) e^{-\tau_{1} t} \int M_{0}^{-1 / 2} f_{0} d x d v
$$

where $\tau_{1}$ is defined in (3).

N.B. The only unspecified dependance of the constants in the two previous results is the ones with respect to $d$ and $n$ which are supposed to be fixed.

\section{Witten Laplacian and rate of convergence}

The lower bound (part 3) of Theorem 2.1) and upper bound (part 4) of Theorem 2.1) for $\tau$ can be specified under additional assumptions by analyzing the Witten Laplacian $A_{0}\left(V_{\beta}\right)$. The simplest case is the $c_{2}$-convex (resp. $c_{2}$-concave) one:

$$
\forall x \in \mathbb{R}^{d}, \quad \pm \operatorname{Hess} V(x) \geq c_{2} \mathrm{Id} .
$$

The low temperature asymptotics $(\beta \rightarrow \infty)$ crucially depends on the number of local minima of $V$ while high temperature asymptotics $(\beta \rightarrow 0)$ can be carried out under rather general assumptions:

Hypothesis $\mathbf{H 2}+$. The potential $V$ is positive near $\infty$ and satisfies

$\mathbf{n}>1$ : The potential $V$ admits a principal part which is an homogeneous potential of degree $2 n, \mathcal{C}^{\infty}$ and non-vanishing outside $x=0$ : There exist $\delta, 0<\delta<2 n-2$, and for any $\alpha \in \mathbb{N}^{d},|\alpha| \leq 2$, a constant $C_{\alpha}^{\prime}$ so that

$$
\forall x \in \mathbb{R}^{d},\left|\partial_{x}^{\alpha} V(x)-\partial_{x}^{\alpha} V_{0}(x)\right| \leq C_{\alpha}^{\prime}\langle x\rangle^{2 n-|\alpha|-\delta} .
$$

$\mathbf{n}=1$ : There exist two positive constants $C_{2}, \kappa_{2}$ and a function $G \in L^{\infty}\left(\mathbb{R}^{d}\right)$ so that for all $x \in \mathbb{R}^{d}$,

$$
\left|\partial_{x} V(x)\right|^{2} \geq C_{2}^{2}|x|^{2}+G(x) \text { and } H e s s V(x)+\frac{1}{2}\left(d C_{2}-\Delta V\right) I d \geq \kappa_{2}^{-1} I d .
$$

Hypothesis H2-. The potential $V$ is negative near $\infty$ and satisfies

$\mathbf{n}>1$ : There exist $n^{\prime}, 1<n^{\prime}<n$, and a constant $C_{2}>0$ so that

$$
\forall x \in \mathbb{R}^{d}, \Delta V(x) \leq C_{2}\left(|x|^{2 n^{\prime}-2}+1\right)
$$

$\mathbf{n}=1$ : There exist two positive constants $C_{2}, \kappa_{2}$ and a function $G \in L^{\infty}\left(\mathbb{R}^{d}\right)$ so that for all $x \in \mathbb{R}^{d}$,

$$
\left|\partial_{x} V(x)\right|^{2} \geq C_{2}^{2}|x|^{2}+G(x) \text { and } d C_{2}-\Delta V(x) \geq 2 \kappa_{2}^{-1} .
$$




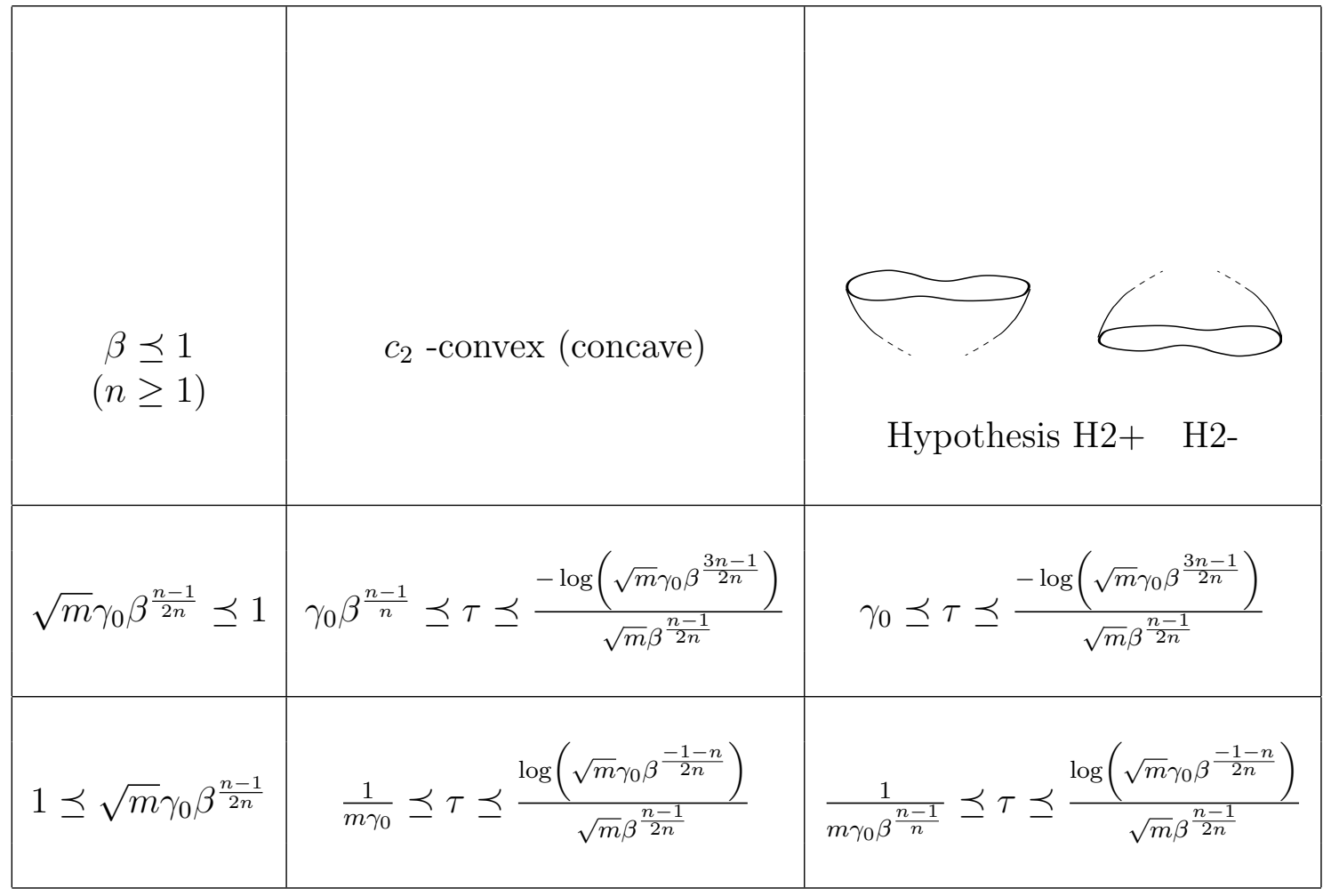

Table 1: High temperature asymptotics.

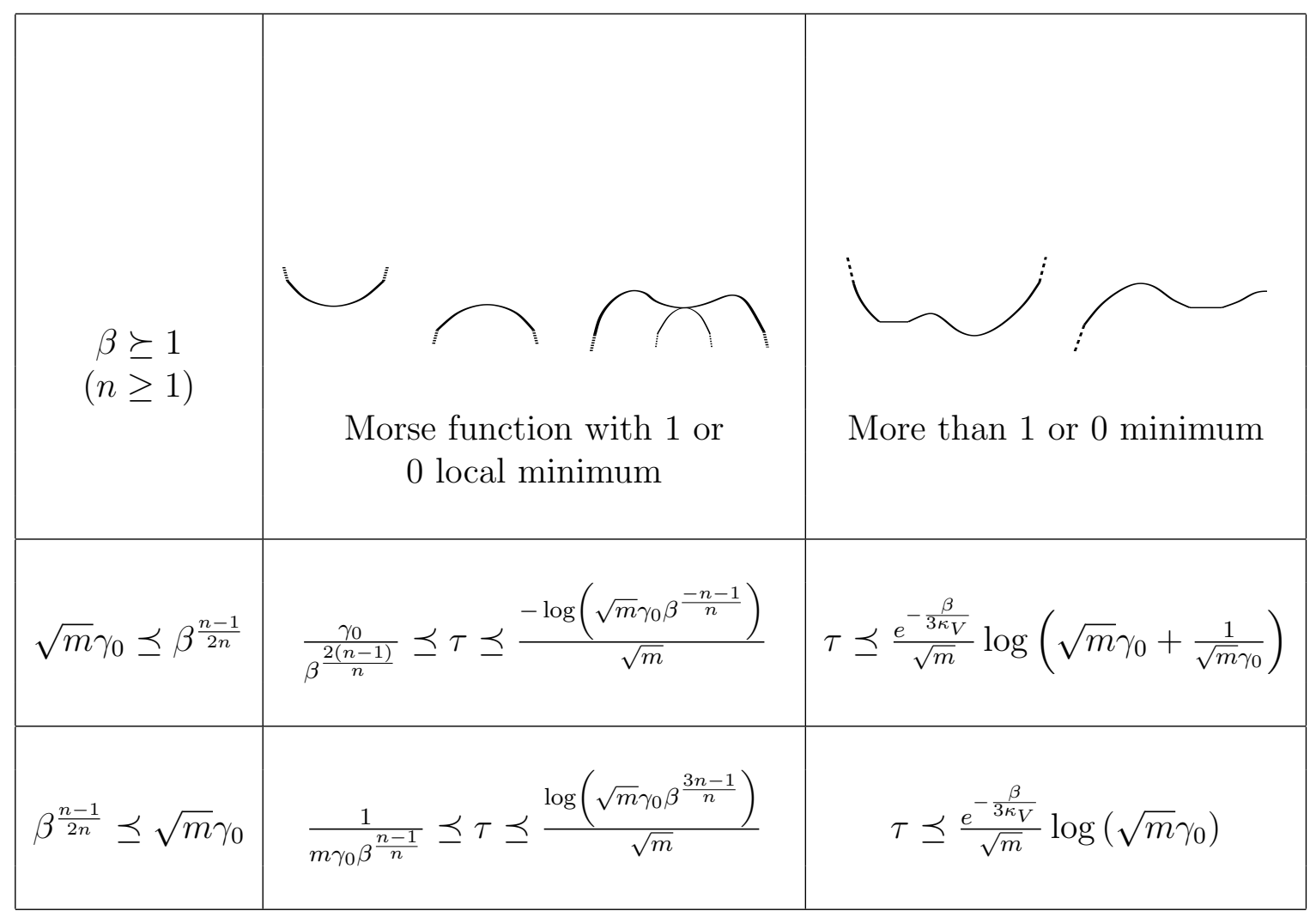

Table 2: Low temperature asymptotics.

The notation $a \preceq b$ means $a \leq \kappa_{V} b$ where $\kappa_{V}$ depends only on $V$. 
Note that things are completely known in the case of a quadratic potential by low-energy approximation of the spectrum (see [24]) or by expressing the solution to (1) with a Mehler formula ([14]). And this says that our lower bounds are optimal in the case $n=1$.

\section{Isotropic hypoellipticity}

After congugating by $M^{1 / 2}$ and scaling, we can write the Fokker-Planck in the more convenient following form (for an Hilbert-space type analysis)

$$
\begin{array}{ll} 
& \partial_{t} u+K u=0 \\
\text { with } & K=v \cdot \partial_{x}-\partial_{x} V_{\beta} \cdot \partial_{v}+\gamma\left(-\partial_{v}+v / 2\right) \cdot\left(\partial_{v}+v / 2\right) \\
\text { and } & V_{\beta}=\beta V\left(\beta^{-\frac{1}{2 n}}\right), \quad \gamma=\gamma_{0} \sqrt{m} \beta^{\frac{n-1}{2 n}}
\end{array}
$$

In the expression of operator $K$ we recognize a transport part

$$
X_{0}=v \cdot \partial_{x}-\partial_{x} V_{\beta} \cdot \partial_{v}
$$

and a diffusion part which is up to a multiplication by $\gamma$ the harmonic oscillator in the velocity variable. The last one can be written with the help of the classical annihilation and creation operators

$$
b=\partial_{v}+v / 2=\left(\begin{array}{l}
\partial_{v_{1}}+v_{1} / 2 \\
\vdots \\
\partial_{v_{d}}+v_{d} / 2
\end{array}\right), \quad b^{*}=\left(\partial_{v_{1}}+v_{1} / 2, \cdots, \partial_{v_{d}}+v_{d} / 2\right),
$$

and we get the following expression for operator $K$

$$
K=X_{0}+b^{*} b .
$$

Operator $K$ is nor selfadjoint neither elliptic. Nevertheless the form vector field + square of first order operator suggests as in [5] and in the spririt of the theorem about sum of squares of vector fields of Hörmander the introduction of the following operators

$$
\begin{gathered}
\gamma^{-1 / 2} a \stackrel{\text { def }}{=} \gamma^{-1 / 2}\left[b, X_{0}\right]=\partial_{x}+\partial_{x} V / 2=\left(\begin{array}{l}
\partial_{x_{1}}+\partial_{x_{1}} V / 2 \\
\vdots \\
\partial_{v_{d}}+\partial_{x_{d}} V / 2
\end{array}\right), \\
\gamma^{-1 / 2} a^{*}=\left(\partial_{x_{1}}+\partial_{x_{1}} V / 2, \cdots, \partial_{x_{d}}+\partial_{x_{1}} V / 2\right),
\end{gathered}
$$

and (say for $\delta>0$ )

$$
\Lambda^{2}=a^{*} a+b^{*} b+\delta .
$$

Operator $\Lambda^{2}$ is formally selfadjoint and elliptic (in an adapted metric). It appears as the sum of the harmonic oscillator $b^{*} b$ and the so-called Witten laplacian

$$
a^{*} a=\gamma\left(-\Delta_{x}+\left(\partial_{x} V\right)^{2} / 4-\Delta V / 2\right) .
$$


The spectral study of operator the Witten laplacean $a^{*} a$ and therefore $\Lambda^{2}$ is well studied (see for example [10, 15]. It is essentially selfadjoint, and under assumptions H1 with compact resolvant and with $\mathcal{S}$ as a core. The strong relations between $K$, and $\Lambda^{2}$ where already known at least in simple cases [24]. Indeed some nice algebraic relations appear such as

$$
\begin{gathered}
-\operatorname{Hess}_{\beta} b=\left[a, X_{0}\right] \\
\text { and } \quad\left[\Lambda^{2}, X_{0}\right]=-b^{*}\left(\operatorname{Hess} V_{\beta}-I d\right) a-a^{*}\left(\operatorname{Hess} V_{\beta}-I d\right) b .
\end{gathered}
$$

The most readable link between operators $K$ and $\Lambda^{2}$ is the following isotropic hypoelliptic estimate for $n>1 / 2, \delta=1$ and $\varepsilon=\min \{1 / 4,1 /(4 n-2)\}$ :

$$
\forall u \in \mathcal{S}\left(\mathbb{R}^{2 d}\right),\left\|\Lambda^{\varepsilon} u\right\|^{2} \leq C_{\text {hyp }}\left(\|K u\|^{2}+\|u\|^{2}\right) .
$$

The choice of $\varepsilon$ comes from a $\psi d o$ analysis inspired by [5], in a metric associated to the Sobolev chain

$$
\mathbb{H}^{r}=\left\{u \in L^{2}, \quad \Lambda^{r} u \in L^{2}\right\} \quad\left(\text { dual } \mathbb{H}^{-r}\right) .
$$

In this calculus, $\Lambda^{2}, K$ and $X_{0}$ are of order 2 whereas $a, b$ and $\partial_{x} V$ are of order 1 . The gain of $\varepsilon$ comes essentially from the fact that $K$ is a differential operator with coefficient with polynomial behavior (at least for the first derivatives) : indeed the commutator $\left[\Lambda^{2}, X_{0}\right]$ is of order $3-\varepsilon$ and not only 3 . In fact the theorem can be deduced from a series of hypoelliptic lemmas that we give below.

As a consequence we get some spectral properties of operator $K$, a priori defined as a differential operator on $\mathcal{S}$ and $\mathcal{S}^{\prime}$. For $r \in \mathbb{R}$, we define the close operators $K_{r}$ with domain

$$
D\left(K_{r}\right)=\left\{u \in \mathbb{H}^{r}, \quad K u \in \mathbb{H}^{r}\right\} .
$$

For all $r$, we get that $\mathcal{S}$ is a core for $K_{r}$, that there is a constant $\lambda_{r}$ depending algebraically on the physical constant such that $\lambda_{r}+K_{r}$ is maximal accretive, and also that $K_{r}$ is with compact resolvant. Besides all $K_{r}$ have the same eigenvalues and eigenfunctions which moreover belong to $\mathcal{S}$. We remove the subscript $r$ for the following.

\section{Some ideas about (pseudo-)spectral estimates}

The Fokker Planck equation in the form $\partial_{t} u+K u$ is of parabolic degenerate type. To prove the properties of the solutions, we want to give a sense to the following Dunford integral (see a.e. [20] [3, pp 556-577] for a general introduction)

$$
e^{-t K}=\frac{1}{2 i \pi} \int_{\Gamma} e^{-t z}(z-K)^{-1} u d z .
$$

Some problems arise in this formula. It supposes that we have a good description of the spectrum of $K$, in order to make the integral converging, and also that uniform bounds for the norm of the resolvant are known (so-called pseudospectral estimates). Let us mention that a priori the resolvant is not a pseudodifferential operator since 
$K$ is not elliptic, and that the numerical range of $K$ is the full right half complex plane.

(Pseudo-)spectral estimates will be partly consequences of the key hypoelliptic estimates of the following type

$$
\begin{array}{r}
\left\|\Lambda^{\varepsilon} u\right\|^{2} \leq \Re \mathrm{e}\left(K u,\left(L+L^{*}\right) u\right)+\Re \mathrm{e}(L K u, L u) \\
+C_{\text {hyp }}^{\prime}\left(\Re \mathrm{e}(K u, u)+\|u\|^{2}\right) .
\end{array}
$$

for $u \in \mathcal{S}$ and where $L=\Lambda^{2 \varepsilon-2} a^{*} b$ and $\varepsilon=\min \{1 / 4,1 /(4 n-2)\}$. As a consequence we get that the spectrum of $K$ is contained in an infinite cusp

$$
S_{K} \stackrel{\text { def }}{=}\left\{z \in \mathbb{C},|z+1|<C_{\infty}|\Re \mathrm{e} z+1|^{N}, \Re \mathrm{e} z \geq-1 / 2\right\},
$$

of frontier $\Gamma=\Gamma_{1} \cup \Gamma_{\infty}$ and where $N=2 / \varepsilon$ (see the picture below). We mention that $C_{\infty}$ is of the form $C_{\infty}=c Q_{R}(C(V), \beta, \gamma)$.

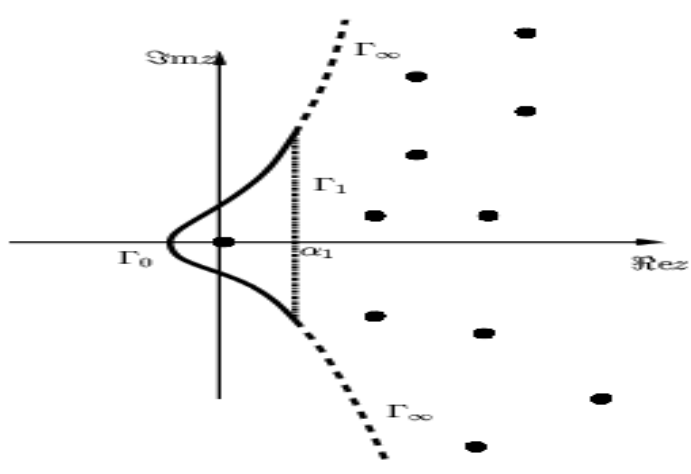

Figure 1: Integration contours: $\partial S_{K}=\Gamma_{0} \cup \Gamma_{\infty}, \partial S_{K}^{\prime}=\Gamma_{1} \cup \Gamma_{\infty}$.

Let us give some ideas about the proof of (11).

1st step : Hypoellipticity and eigenvectors. Let us consider an eigenfunction $u$ associated with an eigenvalue $z=\mu+i \nu \in \mathbb{R}^{+*}+i \mathbb{R}$. the main tool is the estimate (9) applied to $u$ which gives:

$$
\begin{array}{r}
\left\|\Lambda^{\varepsilon} u\right\|^{2} \leq \Re \mathrm{e}\left(z u,\left(L+L^{*}\right) u\right)+\Re \mathrm{e}(L(z u), L u) \\
+C_{\text {hyp }}^{\prime}\left(\Re \mathrm{e}(z u, u)+\|u\|^{2}\right) .
\end{array}
$$


Since $L+L^{*}$ and $L^{*} L$ are symmetric operators we obtain

$$
\left\|\Lambda^{\varepsilon} u\right\|^{2} \leq \mu\left(u,\left(L+L^{*}\right) u\right)+\mu(L u, L u)+C_{\mathrm{hyp}}^{\prime}(\mu+1)\|u\|^{2} .
$$

Now $a \Lambda^{2 \varepsilon-2}$ and its adjoint are bounded operators (by 1). Therefore $\|L u\|^{2}=$ $\left(a \Lambda^{4 \varepsilon-4} a^{*} b u, b u\right) \leq\|b u\|^{2}=\mu\|u\|^{2}$. This yields of course $\mu\|L u\|^{2} \leq \mu^{2}\|u\|^{2}$ and we also obtain

$$
\mu\left(u,\left(L+L^{*}\right) u\right) \leq 2 \mu\|L u\|\|u\| \leq(\mu+1)^{2}\|u\|^{2}
$$

Hence we have

$$
\left\|\Lambda^{\varepsilon} u\right\|^{2} \leq C_{\mathrm{hyp}}^{\prime \prime}(\mu+1)^{2}\|u\|^{2}
$$

for a constant $C_{\text {hyp }}^{\prime \prime}$.

2nd step : functionnal analysis. We want to generalize the estimate

$$
|z+1|^{2}\|u\|^{2} \leq 2\left((K+1)^{*}(K+1) u, u\right)
$$

. Indeed we get for any $\eta \in] 0,1[$,

$$
|z+1|^{2 \eta}\|u\|^{2} \leq 4\left(\left((K+1)^{*}(K+1)\right)^{\eta} u, u\right)
$$

Note that this result is a consequence of a more general result for maximal accretive operator ([12, Appendix B].

3rd step : $\Psi d o$ calculus and functional analysis. From the $\Psi$ do calculus we know that $1 \leq(K+1)^{*}(K+1) \leq C \Lambda^{4}$. Hence we have with $\varepsilon \leq 1 / 4 \leq 1$

$$
\left(\left((K+1)^{*}(K+1)\right)^{\varepsilon / 2} u, u\right) \leq C^{\varepsilon / 2}\left(\Lambda^{2 \varepsilon} u, u\right) .
$$

Synthesis : locus of the spectrum. From steps 1,2 with $\eta=\varepsilon / 2$ and 3 , we can write

$$
\begin{aligned}
\frac{1}{4}|z+1|^{\varepsilon}\|u\|^{2} \leq\left(\left((K+1)^{*}(K+1)\right)^{\varepsilon / 2} u, u\right) & \leq \quad C^{\varepsilon / 2}\left(\Lambda^{2 \varepsilon} u, u\right) \\
& \leq C^{\varepsilon / 2} C_{\text {hyp }}^{\prime \prime}(\mu+1)^{2}\|u\|^{2}
\end{aligned}
$$

and the estimate follows.

Remark 5.1 Actually what is needed for the use of the Dunford integral is a pseudospectral estimate. This can be carried out by using the $\psi$-do calculus and taking $K u=z u+(K-z) u$ instead of $K u=z u$ in the three preceding steps. As a consequence we get on the complement of some changed $S_{K}$ a uniform bound for the resolvant.

Remark 5.2 For low frequencies, for which estimates imply in particular the lower bound for the rate of convergence to the equilibrium, some hypoelliptic results of 
the form (9) are also needed. We bound from below the smallest positive real part of the eigenvalues of $K$ by some constant times the smallest positive eigenvalue of $\Lambda^{2}$. In the computations optimize the parameter $\delta$ in the definition of $\Lambda^{2}$ and strongly use the orthogonal decomposition

$$
K=\left.K\right|_{E_{0}^{\perp}}+\left.K\right|_{E_{0}}
$$

where $E_{0}=\operatorname{Span}\left(M_{0}^{1 / 2}\right)$ when $V$ is positive at infinity. Besides the upper bound is obtained by using quasimodes.

\section{Comments.}

Our approach mimicks Hörmander's method for the operators $c+X_{0}+\sum_{j=1}^{N} X_{j}^{*} X_{j}$ in [13] and its refined version by Kohn in [16]. After the work of Rotschild and Stein [25] the most developed analysis was carried out by Helffer and Nourrigat in [9] and [19] for arbitrary polynomials of vector fields and even polynomials of pseudodifferential operators. Short introductions to this work can be found in [17][18]. We recall that the strategy after the Rotschild-Stein Lifting theorem consists in studying representation of some nilpotent Lie algebra associated with the $X_{k}$ 's. This supposes that the $i X_{k}$ 's are imaginary vector fields or, up to some lower order remainder, symmetric pseudo-differential operators. In our analysis, this is not the case for the annihilation-creation operators $i b$ and $-i b^{*}$. Of course by adding a variable $\theta$, one gets the imaginary vector field $\pm i \partial_{v}-i v \partial_{\theta}$ but no unitary representation allows to go back to the initial situation. Hence there are two options for hypoelliptic problems: 1) Work with symmetric operators $\left(-i \partial_{v}\right.$ and $v$ in our case) and refer to the general theory; 2) Keep non symmetric operators and write adapted commutator estimates. In any of these two cases, applications to pseudospectral estimates rely on a variant of hypoelliptic estimates with symmetric factors of the operator $K$ like in Lemma 9. As previous applications of hypoelliptic techniques, the work of Helffer and Morame [7][8][6] as well as the recent work [4] of Eckmann and Hairer on Fokker-Planck equations follow the first approach. To our knowledge the only work where the non real case was developed is the article [5] of Eckmann, Pillet and Rey-Bellet on Fokker-Planck type equation with short-range perturbations of quadratic potentials. We chose initially this second approach because we thought that the compactness result for the resolvent had nothing to do with choice of coordinate axes in the phase-space, which is contained in the splitting of $i b$ into $-i \partial_{v}$ and $v$. thanks to the nice algebraic properties of the Fokker-Planck equation, we were able to develop the low energy analysis which provides the bounds for the rate of convergence to equilibrium. It is not obvious that such accurate analysis of the low energy spectrum could be done without them.

Beside the nonlinear problems where some other structures given by thermodynamical quantities might enter, possible developments of this work in the linear case may be the study of the Fokker-Planck equation with periodic potential, the case of variable friction, or other degenerate parabolic type equations coming from statistical mechanics ([5]) or from linearized kinetic equations (see the survey paper [28]). 
Eventually a recent answer to the conditions insuring the compactness of the resolvant of the Witten Laplacian has been given in [11]. As a consequence it gives necessary conditions on the possible degeneracy at infinity of the potential leading to exponential trend to the equilibrium. Meanwhile our proof has to be adapted to this case.

\section{References}

[1] F. Bouchut and F. Dolbeault. On long time asymptotics of the Vlasov-FokkerPlanck equation and of the Vlasov-Poisson-Fokker-Planck system with Coulombic and Newtonian potentials. Differential and Integral Equations, 8(3):487$514,1995$.

[2] L. Desvillettes and C. Villani. On the trend to global equilibrium in spatially inhomogeneous entropy-dissipating systems: the linear Fokker-Planck equation. Comm. Pure Appl. Math., 54(1):1-42, 2001.

[3] N. Dunford and J.T. Schwartz. Linear operators. Part I. John Wiley \& Sons Inc., New York, 1988.

[4] J.P. Eckmann and M. Hairer. Non-equilibrium statistical mechanics of strongly anharmonic chains of oscillators. Comm. Math. Phys., 212(1):105-164, 2000.

[5] J.P. Eckmann, C.A. Pillet, and L. Rey-Bellet. Non-equilibrium statistical mechanics of anharmonic chains coupled to two heat baths at different temperatures. Comm. Math. Phys., 201(3):657-697, 1999.

[6] B. Helffer. Semi-classical analysis for the Schrödinger operator and applications, volume 1336 of Lect. Notes in Mathematics. Springer-Verlag, Berlin, 1988.

[7] B. Helffer and A. Mohamed. Caractérisation du spectre essentiel de l'opérateur de Schrödinger avec un champ magnétique. Ann. Inst. Fourier (Grenoble), 38(2):95-112, 1988.

[8] B. Helffer and A. Mohamed. Semiclassical analysis for the ground state energy of a Schrödinger operator with magnetic wells. J. Funct. Anal., 138(1):40-81, 1996.

[9] B. Helffer and J. Nourrigat. Hypoellipticité maximale pour des opérateurs polynômes de champs de vecteurs. Birkhäuser Boston Inc., Boston, MA, 1985.

[10] B. Helffer and J. Sjöstrand. Puits multiples en mécanique semi-classique. IV. étude du complexe de Witten. Comm. Partial Differential Equations, 10(3):245-340, 1985.

[11] B. Helffer and F. Nier. work in preparation.

[12] F. Hérau and F. Nier. Isotropic hypoellipticity and trend to the equilibrium for the Fokker-Planck equation with high degree potential. preprint University of Rennes 1, 2002. 
[13] L. Hörmander. Hypoelliptic second order differential equations. Acta Math., 119:147-171, 1967.

[14] L. Hörmander. Symplectic classification of quadratic forms, and general Mehler formulas. Math. Z., 219(3):413-449, 1995.

[15] J. Johnsen. On the spectral properties of Witten-Laplacians, their range projections and Brascamp-Lieb's inequality. Integral Equations Operator Theory, 36(3):288-324, 2000.

[16] J.J. Kohn. Lectures on degenerate elliptic problems. In Pseudodifferential operator with applications ( CIME 1977), pages 89-151. Liguori, Naples, 1978.

[17] G. Métivier. Équations aux dérivées partielles sur les groupes de Lie nilpotents. In Bourbaki Seminar, Vol. 1981/1982, pages 75-99. Soc. Math. France, Paris, 1982 .

[18] J. Nourrigat. Systèmes sous-elliptiques. In Séminaire sur les équations aux dérivées partielles 1986-1987, pages Exp. No. V, 14. École Polytech., Palaiseau, 1987.

[19] J. Nourrigat. Systèmes sous-elliptiques. II. Invent. Math., 104(2), 1991.

[20] M. Reed and B. Simon. Methods of Modern Mathematical Physics, volume 2. Acad. Press, 1975.

[21] L. Rey-Bellet and L.E. Thomas. Asymptotic Behavior of Thermal Nonequilibrium Steady States for a Driven Chain of Anharmonic Oscillators. Comm. Math. Phys., 215:1-24, 2000.

[22] L. Rey-Bellet and L.E. Thomas. Exponential Convergence to Non-Equilibrium Stationary States in Classical Statistical Mechanics. Comm. Math. Phys., 225:305-329, 2000.

[23] L. Rey-Bellet and L.E. Thomas. Fluctuations of the Entropy Production in Anharmonic Chains. preprint 2002.

[24] H. Risken. The Fokker-Planck equation. Springer-Verlag, Berlin, second edition, 1989. Methods of solution and applications.

[25] L.P. Rothschild and E.M. Stein. Hypoelliptic differential operators and nilpotent groups. Acta Math., 137(3-4):247-320, 1976.

[26] D. Talay. Approximation of invariant measures of nonlinear Hamiltonian and dissipative stochastic differential equations. In C. Soize R. Bouc, editor, Progress in Stochastic Structural Dynamics, volume 152 of Publication du L.M.A.-C.N.R.S., pages 139-169, 1999.

[27] D. Talay. Stochastic Hamiltonian dissipative systems with non globally Lipschitz coefficients: exponential convergence to the invariant measure and discretization by the implicit Euler scheme. to appear in Markov Processes and Related Fields. 
[28] C. Villani. A review of mathematical topics in collisional kinetic theory. to appear in Handbook of Fluid Mechanics S. Friedlander and D. Serre Eds.

IRMAR, Campus de Beaulieu, Université De Rennes 1, 35042 Rennes Cedex, France

fherau34@axmel. net

www.maths.univ-rennes1.fr $/ \sim$ fherau 